\title{
Análise espacial da mortalidade neonatal precoce no Município do Rio de Janeiro, 1995-1996
}

\author{
Spatial analysis of early neonatal mortality \\ in the municipality of Rio de Janeiro, 1995-1996
}

Carla Lourenço Tavares de Andrade 1

Célia Landmann Szwarcwald 1

\footnotetext{
1 Departamento de Informações em Saúde, Centro de Informação Científica e Tecnológica, Fundação Oswaldo Cruz Av Brasil 4365, Rio de Janeiro, $R J$ 21045-900, Brasil. carlamv@fiocruz.br
}

\begin{abstract}
The objective of this study was to establish the spatial distribution of the early neonatal mortality rate (0-3 days) in the municipality of Rio de Janeiro for 1995-1996, identifying the best explanatory factors for spatial variations. By considering Rio de Janeiro's 153 neighborhoods as ecological units of analysis, socioeconomic and maternal indicators were analyzed according to place of residence. Spatial statistical analysis was performed using the Cliff \& Ord methodology, appropriate for lattice data. From the 0-3 day mortality thematic map, we clearly identified two clusters of high early neonatal mortality rates. Spatial dependence was also confirmed by the statistical results of the spatial analysis. The variables that explain the clusters are the proportions of "adolescent mothers", "slum-dwellers", and "heads of households below the poverty level". Spatial statistics provided a better understanding of the geographic distribution of early neonatal mortality and suggested potential hypotheses for further investigation, which could support preventive programs and contribute to a decrease in infant mortality.
\end{abstract}

Key words Spatial Analysis; Neonatal Mortality; Pregnancy in Adolescent

Resumo O objetivo deste trabalho foi estabelecer a distribuição espacial da mortalidade neonatal precoce (0-3 dias) no Município do Rio de Janeiro de 1995 a 1996, visando identificar os fatores mais explicativos das variações espaciais. Considerando os bairros como unidade ecológica de análise, foram analisados indicadores relativos às condições sócio-econômicas e às características das mães dos recém-nascidos por bairro de residência. A análise estatística espacial dos dados foi realizada utilizando as técnicas de Cliff \& Ord, apropriadas para "dados de áreas". Por meio do mapa temático da mortalidade de 0-3 dias, observou-se nitidamente a presença de dois aglomerados de taxas elevadas. A dependência espacial foi igualmente constatada pelos resultados da análise estatística. As variáveis que melhor explicaram os aglomerados espaciais foram: "proporção de mães adolescentes", "proporção de pessoas residentes em favelas em 1996" e "proporção de chefes com rendimento até um salário mínimo". A metodologia de estatística espacial permitiu maior compreensão da distribuição geográfica da mortalidade neonatal precoce, e deu margem a outros tipos de investigações, que poderão subsidiar os programas preventivos e contribuir ao declínio da mortalidade infantil.

Palavras-chave Análise Espacial; Mortalidade Neonatal; Gravidez na Adolescência 


\section{Introdução}

No Brasil, vários estudos têm demonstrado a tendência de declínio da mortalidade infantil (Menezes et al., 1996). Entretanto, a evolução de decréscimo não é homogênea no país, com um padrão que obedece às desigualdades regionais e, sobretudo, à iniqüidade das condições sócio-econômicas (Menezes et al., 1998).

Diferenças importantes também são encontradas por grupo etário. Enquanto o decréscimo do componente pós-neonatal é nítido em todas as regiões do país, trabalhos recentes mostram que a mortalidade neonatal tem apresentado pouco declínio ou estabilidade como, por exemplo, nos casos dos estados do Rio Grande do Sul (SSMA-RS, 1998) e do Rio de Janeiro (Leal \& Szwarcwald, 1996).

Adicionalmente, estudos recentes têm relacionado o comportamento evolutivo dos indicadores de saúde, dentre eles a mortalidade infantil, à distribuição de renda da população, enfatizando a relevância da pobreza relativa frente à frustração provocada pela exclusão social e material das oportunidades proporcionadas pela sociedade (Kaplan, 1996; Wilkinson, 1997).

A acentuação da desigualdade na distribuição de renda de vários países foi acompanhada por um importante crescimento na concentração residencial da pobreza (Kawachi \& Kennedy, 1997), aumentando particularmente as disparidades sociais nas grandes cidades e, em paralelo, as variações intra-urbanas das condições de saúde (Massey, 1996).

No que diz respeito às diferenças da mortalidade infantil frente às desigualdades sócioeconômicas nas grandes cidades, pesquisas realizadas em diversos países têm identificado grandes variações no padrão deste indicador nos centros urbanos. Rip et al. (1987), em estudo realizado na área metropolitana da Cidade do Cabo, África do Sul, cujo objetivo foi determinar a variação espacial da mortalidade neonatal no nível do subúrbio dentro da cidade, os resultados demonstraram a hipótese de relação inversa entre o nível sócio-econômico e a taxa de mortalidade infantil. Através da análise espacial, variações na taxa de mortalidade infantil foram significativamente correlacionadas com as variações do nível sócioeconômico.

Issler et al. (1996), no Brasil, em estudo de população urbana de uma área específica da cidade Porto Alegre, teve o objetivo de testar a hipótese de que, em uma população de baixo nível sócio-econômico, diferentes níveis de pobreza podem acarretar distintas condições de saúde. Os autores mostraram que, entre as famílias de extrema pobreza, as crianças apresentaram maior taxa de mortalidade infantil e menor peso ao nascer, identificando assim subgrupos da população que necessitam de ações de saúde específicas.

No caso particular do Município do Rio de Janeiro, acompanhando o aumento da desigualdade na distribuição de renda no país nas últimas duas décadas, houve expansão relevante de comunidades faveladas, que se concentraram geograficamente em certas áreas da cidade (Corrêa, 1996).

Este trabalho tem o objetivo principal de realizar uma análise espacial da mortalidade durante os primeiros dias de vida, no Município do Rio de Janeiro, visando estabelecer a distribuição espacial da taxa de mortalidade neonatal precoce e identificar quais os principais fatores responsáveis pelas variações espaciais. Com este fim, os bairros da cidade são considerados como unidade ecológica de análise e as variações espaciais da taxa de mortalidade nos três primeiros dias de vida são estudadas frente a alguns indicadores como as condições sócio-econômicas e as características das mães dos recém-nascidos por bairro de residência.

\section{Material e métodos}

Trata-se de um estudo ecológico considerando, como unidades de análise, os 153 bairros do Município do Rio de Janeiro.

A base digital para os bairros utilizada neste trabalho foi gerada no Laboratório de Geoprocessamento do Departamento de Informação em Saúde (DIS), Centro de Informação Científica e Tecnológica (CICT), Fundação Oswaldo Cruz (FIOCRUZ) no âmbito do Projeto SIG-FIOCRUZ.

As fontes de dados foram provenientes de três tipos de sistemas: o de nascidos vivos (SINASC); o Sistema de Informações sobre Mortalidade (SIM) - ambos cedidos gentilmente pela Secretaria Municipal de Saúde do Município do Rio de Janeiro para elaboração deste trabalho - e as informações do Censo Demográfico de 1991 referentes aos chefes de domicílios, fornecidas pela Fundação Instituto Brasileiro de Geografia e Estatística (IBGE, 1994), onde os dados por setor censitário foram agregados por bairros através do SIG-FIOCRUZ.

Escolheu-se a mortalidade de 0 a 3 dias como objeto da presente análise, já que se trata do estudo de óbitos que ocorrem próximo ao momento do parto e que constituem um grupo 
cuja taxa de mortalidade tem apresentado comportamento temporal de pouco declínio ou, até mesmo, de estabilidade nos últimos anos, diferentemente das outras faixas etárias.

Para a análise da mortalidade nos três primeiros dias de vida, a taxa média de mortalidade por mil nascidos vivos (/1.000 NV) foi calculada pela divisão do número de óbitos ocorridos nos três primeiros dias de vida no período 1995-96 pelo número de nascidos vivos no mesmo período. Com o objetivo de evitar viés na estimativa das taxas acarretado por denominadores pequenos, os bairros onde o número de nascidos vivos foi menor do que 400, no período 1995-96, não foram considerados na análise estatística espacial.

A visualização da taxa de mortalidade nos três primeiros dias de vida por bairro do Município do Rio de Janeiro foi feita através de mapa temático elaborado com o software MapInfo (MapInfo Corporation, 1994), definindo-se como ponto de corte um terço das observações para cada faixa.

Os indicadores utilizados para os bairros do Município do Rio de Janeiro estão descritos a seguir.

Indicadores sócio-demográficos

(provenientes do Censo Demográfico, 1991)

a) Proporção de mulheres em idade fértil analfabetas - calculada como a divisão entre o número de mulheres de 15 a 49 anos analfabetas e o total de mulheres de 15 a 49 anos.

b) Proporção de pessoas residentes em favelas em 1996 - calculada como a divisão entre o número de pessoas residentes em favelas e a população total.

c) Densidade de população favelada, por km², em 1996 - calculada como a divisão entre o número de pessoas residentes em favelas e a área do bairro.

d) Proporção de chefes com rendimento até um salário mínimo - calculada como a divisão entre o número de chefes sem rendimento adicionado aos que ganham até um salário mínimo e o número total de chefes (excluídos os sem declaração).

Indicadores provenientes do arquivo de nascidos vivos, 1995 e 1996

a) Proporção de mães adolescentes - calculada como a divisão entre o número de mães de 10 a 19 anos de idade e o número total de mães (excluindo-se as de idade ignorada).

b) Proporção de partos cesáreos - calculada como a divisão entre o número de partos do ti- po cesárea e o número de nascidos vivos, excluindo-se os de tipo de parto ignorado.

c) Coeficiente geral de natalidade, em 1996 - calculado como a divisão entre o número de nascidos vivos e a população total.

d) Proporção de nascimentos em estabelecimentos de saúde privados - calculada como a divisão entre o número de nascidos vivos em hospitais privados e o número de nascidos vivos, excluindo-se os de estabelecimentos ignorados.

e) Proporção de mães que não fizeram prénatal - calculada como a divisão entre o número de mães que não fizeram pré-natal e o número de nascidos vivos, excluindo-se os ignorados.

Para a "taxa de mortalidade nos três primeiros dias de vida", para a "proporção de pessoas residentes em favelas em 1996", para a "densidade de população favelada, por km², em 1996” e para o "coeficiente geral de natalidade, em 1996" foi utilizada a transformação do tipo raiz quadrada. Já para a "proporção de mulheres em idade fértil analfabetas", para a "proporção de chefes com rendimento até um salário mínimo", para a "proporção de mães adolescentes" e para a "proporção de partos cesáreos" foi empregada a transformação do tipo logaritmo neperiano. Estas transformações foram escolhidas porque resultaram em melhores aproximações para a distribuição normal. Não houve nenhum tipo de transformação para os indicadores "proporção de nascimentos em estabelecimentos de saúde privados" e "proporção de mães que não fizeram pré-natal”.

Preliminarmente, para descrever a associação entre todas as variáveis estudadas, foi construída a matriz de correlações, através do software SPSS (SPSS Incorporation, 1995).

A análise estatística espacial dos dados foi realizada utilizando as técnicas de Cliff \& Ord (1981), apropriadas para "dados de áreas" (lattice data). Esta metodologia é adequada quando se deseja estudar a distribuição espacial de algum fenômeno como, no nosso exemplo, a taxa de mortalidade nos três primeiros dias em uma base cartográfica predefinida, e testar a hipótese de independência espacial dos dados. Como medidas da dependência espacial, são utilizados coeficientes de autocorrelação espacial, como o coeficiente I de Moran (1950) ou o coeficiente c de Geary (1954).

No caso da presente análise, para mensurar a autocorrelação espacial da taxa de mortalidade nos três primeiros dias de vida, utilizouse o coeficiente de Moran dado por: 
$\mathrm{I}=\frac{n \sum_{i} \sum_{j} w_{i j} z_{i} z_{j}}{S_{0} \sum_{i} z_{i}^{2}}$

$i, j=1, \ldots, 153$ bairros

onde:

$S_{0}=\sum_{i} \sum_{j} w_{i j}$

$z_{i}=x_{i}-\bar{x}$

$\mathrm{x}_{\mathrm{i}}=$ taxa de mortalidade nos três primeiros dias de vida

$\mathrm{w}_{\mathrm{ij}}=$ ponderação espacial atribuída ao par de bairros (i,j)

Este índice é entendido como um coeficiente de correlação entre valores da mesma variável mensurada em locais vizinhos i e j. Varia, em geral, entre - 1 e 1, embora possam ocorrer exceções. Para testar o coeficiente de autocorrelação, a hipótese nula é definida como a de não correlação e a hipótese alternativa, por uma matriz de ponderações espaciais $\left(\mathrm{w}_{\mathrm{ij}}\right)$, que expressam a proximidade entre as áreas geográficas e podem ser definidas por meio da contiguidade, da distância ou de quaisquer outros critérios de vizinhança entre as localidades.

Demonstra-se que I tem uma distribuição assintótica normal (Sen, 1990), e, portanto, utiliza-se a aproximação normal para o coeficiente I padronizado com a finalidade de testar a hipótese de ausência de autocorrelação espacial.

O cálculo da média e variância do coeficiente I pode ser feito sob duas suposições: normalidade (N) ou aleatoriedade (R). Na primeira, os dados são observações independentes de uma distribuição normal. Já na segunda, o método não assume nenhuma distribuição específica (Cliff \& Ord, 1981). Desse modo, a média e variância sob as duas suposições são assim definidas:

$E_{N}(I)=E_{R}(I)=\frac{-1}{n-1}$

$\operatorname{Var}_{N}(I)=\frac{n^{2} S_{1}-n S_{2}+3 S_{0}^{2}}{S_{0}^{2}\left(n^{2}-1\right)}$

$\operatorname{Var}_{R}(I)=\frac{n\left[\left(n^{2}-3 n+3\right) S_{1}-n S_{2}+3 S_{0}^{2}\right]-b_{2}\left[\left(n^{2}-n\right) S_{1}-2 n S_{2}+6 S_{0}^{2}\right]}{(n-1)^{3} S_{0}^{2}}$

onde:

$$
S_{0}=\sum_{i} \sum_{j} w_{i j}
$$

$S_{1}=\frac{1}{2} \sum_{i} \sum_{j}\left(w_{i j}+w_{j i}\right)^{2}$

$S_{2}=\sum_{i}\left(w_{i .}+w_{. i}\right)^{2}$

onde:

$w_{. i}=\sum_{j} w_{j i}$

$w_{i .}=\sum_{j} w_{i j}$

Na presente análise, a variável escolhida para objeto de investigação espacial foi a raiz quadrada da taxa de mortalidade nos três primeiros dias de vida, cuja aproximação para a distribuição normal foi bastante satisfatória (Figura 1). A análise espacial foi feita utilizando-se o software S-Plus (Mathsoft, 1996).

Para que o coeficiente I de Moran fosse calculado, foi necessária a construção de uma matriz de vizinhança para os 153 bairros do Município do Rio de Janeiro. Esta foi definida por bairros contíguos, com pelo menos um ponto em comum. As ponderações $\mathrm{w}_{\mathrm{ij}}$ foram consideradas como o inverso da distância entre os centróides dos bairros $i$ e $j$.

Para testar a hipótese de independência espacial, o teste de significância de I foi feito a partir da suposição de normalidade das observações, calculando-se o índice padronizado como:

$I_{p a d r}=\frac{I-E(I)}{\sqrt{\operatorname{Var}(I)}}$

onde a média e a variância são calculadas através das fórmulas (1) e (2)

que tem distribuição assintótica normal padrão (Sen, 1990).

No caso de significância estatística do coeficiente $\mathrm{I}_{\text {padr }}$ de autocorrelação espacial para a variável relativa à taxa de mortalidade nos três primeiros dias de vida, as variáveis independentes consideradas foram estudadas quanto à sua associação aos conglomerados espaciais encontrados.

Em semelhança aos modelos de regressão múltipla utilizados para seleção das variáveis mais relevantes à explicação da resposta, o procedimento empregado para escolher os fatores mais explicativos dos conglomerados espaciais foi um procedimento passo a passo, proposto por (Anselin, 1992), baseado em minimizar o coeficiente $\mathrm{I}_{\text {padr }}$, calculado por meio dos resíduos da regressão da variável resposta com as variáveis independentes, até que este perca a sua significância. 
A idéia intuitiva deste procedimento está em apontar quais as variáveis que mais explicam a dependência espacial da resposta, no sentido de que, controlados os efeitos destas variáveis mediante os resíduos do modelo de regressão, a autocorrelação espacial perderia a sua significância estatística inicial.

A aplicação deste procedimento requer o cálculo do coeficiente de autocorrelação I para os resíduos da regressão, que são correlacionados, implicando uma modificação no cálculo da média e da variância do coeficiente I, como demonstrado por Sen (1990).

Neste trabalho, para o uso do procedimento passo a passo foi preciso modificar as estimativas do coeficiente $\mathrm{I}_{\text {padr }}$, elaboradas pelo software S-Plus (Mathsoft, 1996), que não incorpora as alterações dos momentos de I citadas anteriormente. Com tal propósito, foi elaborado um programa em linguagem $\mathrm{C}$, que calcula o coeficiente $\mathrm{I}_{\text {padr }}$ com as devidas correções.

No primeiro passo, realizou-se uma regressão simples entre a variável resposta (raiz quadrada da taxa de mortalidade nos três primeiros dias de vida) e cada variável independente, calculando-se os resíduos correspondentes e o coeficiente de autocorrelação (I) com os resíduos assim obtidos. A primeira variável selecionada como o indicador com maior contribuição à dependência espacial foi aquela cujos resíduos demonstraram o menor $\mathrm{I}_{\text {padr. No se- }}$ gundo passo, repetiu-se o mesmo procedimento, mas a regressão foi composta de duas variáveis independentes, incluindo sempre a variável selecionada no passo 1 . O procedimento foi repetido até obter-se coeficiente $\mathrm{I}_{\text {padr }}$ não significativo, no nível de significância de $10 \%$.

\section{Resultados}

O mapa temático por bairros para a taxa de mortalidade nos três primeiros dias de vida está disposto na Figura 2. Observa-se nitidamente a presença de dois aglomerados. Um, denominado "Aglomerado 1" - composto pelos bairros de Santa Cruz, Guaratiba e Recreio dos Bandeirantes - e o outro, denominado "Aglomerado 2" constituído pelos bairros de Senador Camará, Padre Miguel, Realengo, Ricardo de Albuquerque, Guadalupe, Costa Barros, Barros Filho, Marechal Hermes, Rocha Miranda, Oswaldo Cruz, Turiaçu, Vila Valqueire, Campinho, Tanque, Taquara, Curicica, Cidade de Deus, Vaz Lobo, Vicente de Carvalho, Cavalcanti, Vila Kosmos, Penha Circular, Engenho da Rainha e Pilares.

O primeiro aglomerado concentra-se na Zona Oeste da cidade e a maioria dos bairros en-
Figura 1

Desvios em relação ao gráfico de probabilidade da distribuição normal.
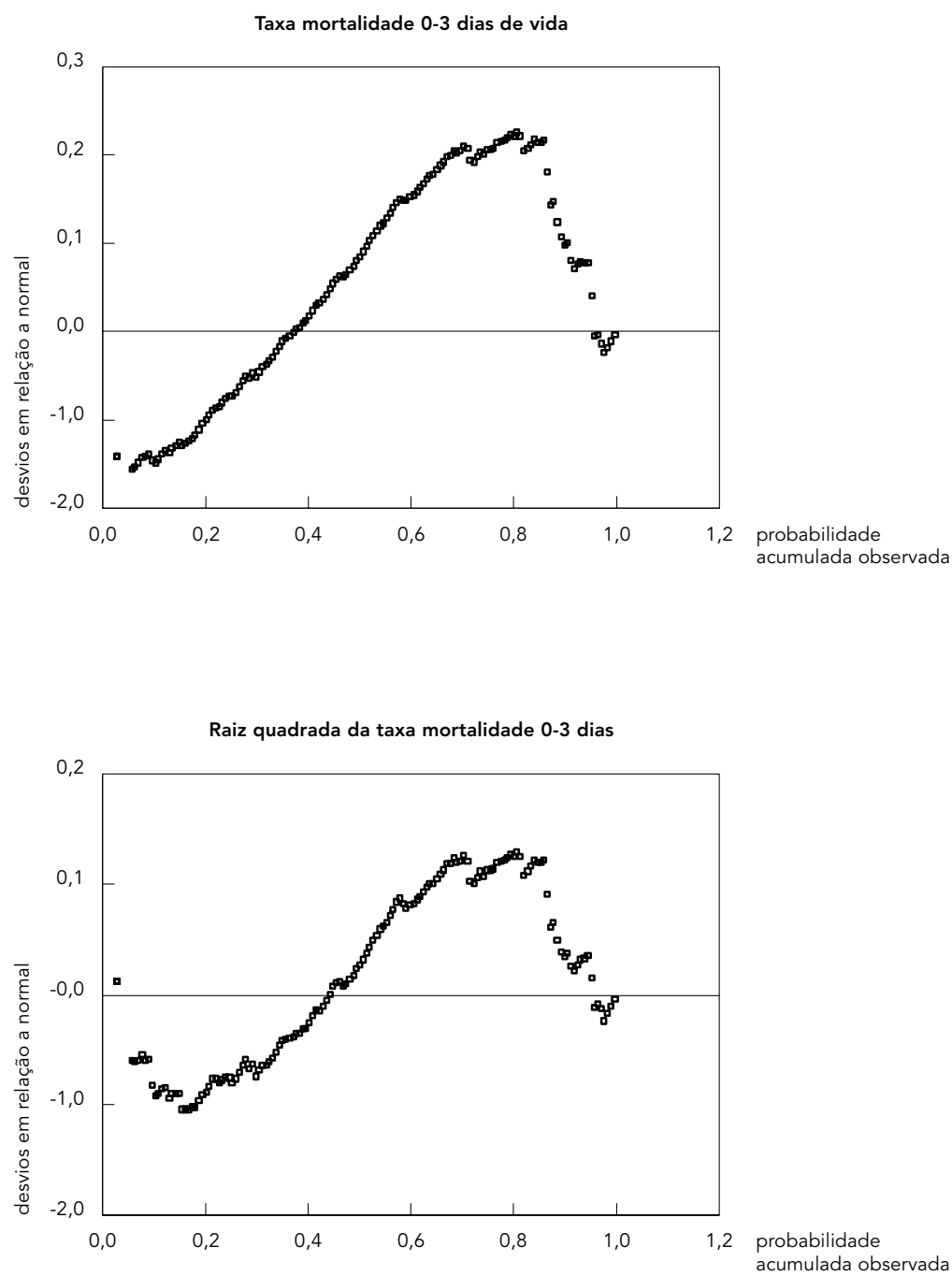

volvidos pertence às regiões administrativas (RA) de Santa Cruz e Guaratiba, áreas, reconhecidamente, de baixo nível sócio-econômico. Diferentemente do primeiro, o segundo conglomerado é disperso e se constitui de bairros das RAs de Bangu, Madureira, Jacarepaguá, Penha, Irajá e Anchieta.

Na Tabela 1, estão dispostos os resultados da análise estatística espacial da variável resposta, a raiz quadrada da taxa de mortalidade nos três primeiros dias de vida. O coeficiente de Moran de autocorrelação espacial é igual a 0,1303 e foi significativo no nível de $1 \%$ (valor de $\mathrm{p}=0,005$ ), indicando ocorrência de depen- 


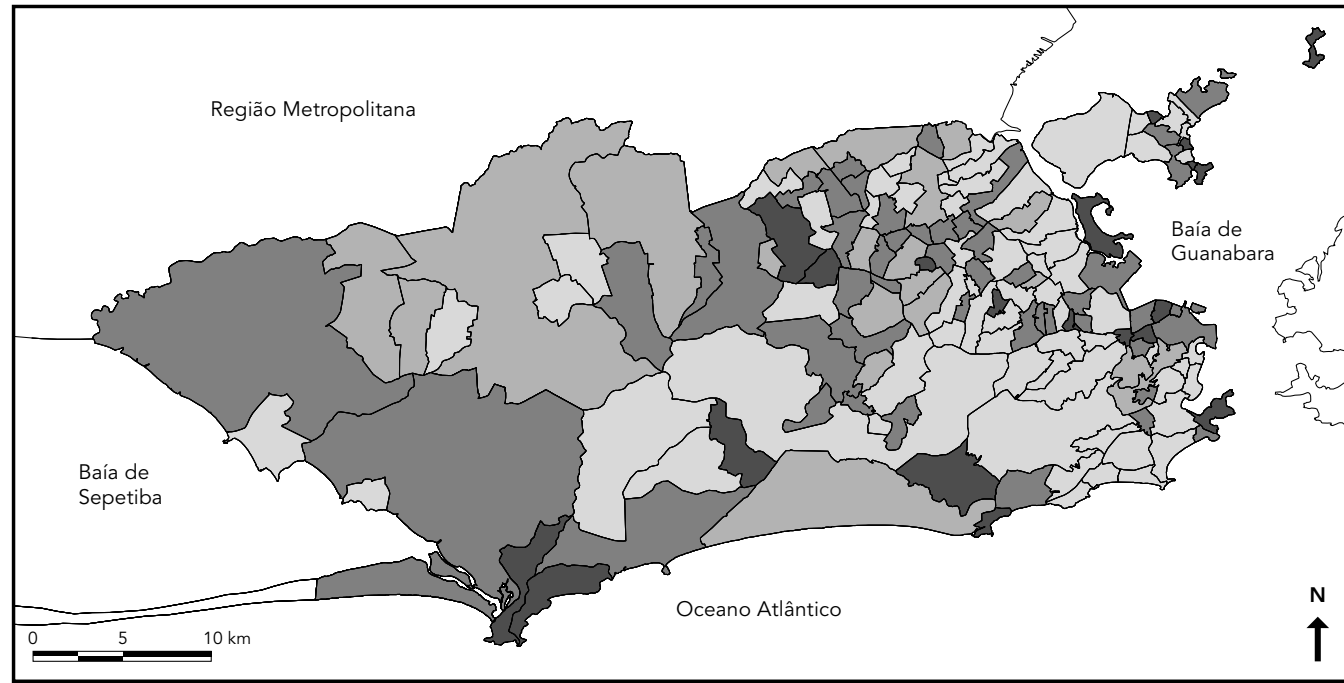

$$
\begin{aligned}
& \text { até } 8,5 \\
& 8,6 \text { a } 10,0 \\
& 10,1 \text { e mais } \\
& \text { bairro }<200 \mathrm{NV}
\end{aligned}
$$

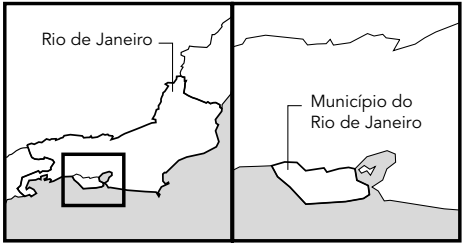

Fonte: Secretaria Municipal de Saúde do Rio de Janeiro e Sistema de Informação Geográfica/Fundação Oswaldo Cruz. $\mathrm{NV}=$ nascidos vivos.

dência espacial e corroborando os achados de conglomerados de taxas elevadas visualizados na Figura 2.

A matriz dos coeficientes de correlação entre todos os indicadores considerados para os bairros do Município do Rio de Janeiro está apresentada na Tabela 2. Os indicadores que são forte e positivamente correlacionados à variável relacionada à mortalidade nos três primeiros dias de vida são a "proporção de mães adolescentes", a "proporção de chefes com rendimento até um salário mínimo" e a "proporção de mães que não fizeram pré-natal”. Evidencia-se, assim, que quanto maior a proporção de chefes com rendimento até um salário mínimo, a proporção de mães adolescentes e a proporção de mães que não fizeram pré-natal, maior é a taxa de mortalidade nos três primeiros dias de vida. Chama a atenção que essas três variáveis são igualmente correlacionadas entre si, constituindo-se em um conjunto de fatores associado ao excesso de óbitos nos primeiros dias de vida.
A proporção de partos cesáreos é a única variável correlacionada inversa e significativamente à mortalidade nos três primeiros dias de vida. Entretanto, a proporção de cesarianas apresenta, por outro lado, associação positiva com a proporção de nascimentos em estabelecimentos privados, o que parece explicar, pelo menos em parte, a associação inversa encontrada com a resposta (Tabela 2).

A partir do procedimento para a seleção da variável mais explicativa dos aglomerados espaciais, tal qual exposto na metodologia, constata-se que a variável "proporção de mães adolescentes” é aquela que tem maior poder de explicação. O coeficiente de Moran, calculado com os resíduos correspondentes a esta variável é o menos significativo, apresentando o maior valor de $\mathrm{p}(\mathrm{I}=0,0917$; $\mathrm{p}=$ 0,08533).

Observa-se que, após a escolha da variável "proporção de mães adolescentes", o coeficiente de autocorrelação padronizado perde a significância estatística no nível de 5\%, e o proce- 
Coeficientes I de Moran, I padronizado e correspondentes níveis descritivos de significância.

\begin{tabular}{|c|c|c|c|}
\hline Sem Ajuste & 1 & I padronizado & $\mathrm{p}$ \\
\hline Raiz quadrada taxa mortalidade 0-3 dias & 0,1303 & 2,7889 & 0,00528 \\
\hline 1 Passo: Taxa mortalidade 0-3 dias ajustada por & 1 & I padronizado & $p$ \\
\hline Proporção de mães adolescentes & 0,0917 & 1,7215 & 0,08533 \\
\hline Proporção chefes com rendimento até um salário mínimo & 0,0970 & 1,8143 & 0,06953 \\
\hline Proporção de mães que não fizeram pré-natal & 0,1307 & 2,4046 & 0,01623 \\
\hline Proporção de partos cesáreos & 0,1379 & 2,5307 & 0,01137 \\
\hline Proporção nascimentos em estabelecimentos privados & 0,1583 & 2,8881 & 0,00387 \\
\hline Proporção de mulheres em idade fértil analfabetas & 0,1626 & 2,9634 & 0,00304 \\
\hline Densidade de população favelada, por km², em 1996 & 0,1661 & 3,0247 & 0,00249 \\
\hline Proporção de pessoas residentes em favelas em 1996 & 0,1707 & 3,1053 & 0,00191 \\
\hline Coeficiente geral de natalidade, em 1996 & 0,1846 & 3,3487 & 0,00081 \\
\hline 2o Passo: Taxa mortalidade 0-3 dias ajustada por "proporção de mães adolescentes" e por: & 1 & I padronizado & p \\
\hline Proporção de pessoas residentes em favelas em 1996 & 0,0892 & 1,6777 & 0,09361 \\
\hline Proporção chefes com rendimento até um salário mínimo & 0,0895 & 1,6829 & 0,09238 \\
\hline Proporção de mulheres em idade fértil analfabetas & 0,0912 & 1,7127 & 0,08694 \\
\hline Proporção de mães que não fizeram pré-natal & 0,0913 & 1,7139 & 0,08655 \\
\hline Densidade de população favelada, por km², em 1996 & 0,0914 & 1,7162 & 0,08617 \\
\hline Proporção de partos cesáreos & 0,0937 & 1,7565 & 0,07892 \\
\hline Proporção nascimentos em estabelecimentos privados & 0,0980 & 1,8318 & 0,06691 \\
\hline Coeficiente geral de natalidade, em 1996 & 0,1165 & 2,1559 & 0,03113 \\
\hline $\begin{array}{l}\text { 3o Passo: Taxa mortalidade 0-3 dias ajustada por "proporção de mães adolescentes", } \\
\text { por "proporção de pessoas residentes em favelas em 1996" e por: }\end{array}$ & 1 & I padronizado & $p$ \\
\hline Proporção chefes com rendimento até um salário mínimo & 0,0822 & 1,5549 & 0,12000 \\
\hline Proporção de mães que não fizeram pré-natal & 0,0888 & 1,6714 & 0,09466 \\
\hline Proporção de mulheres em idade fértil analfabetas & 0,0926 & 1,7365 & 0,08249 \\
\hline Coeficiente geral de natalidade, em 1996 & 0,0946 & 1,7714 & 0,07652 \\
\hline Proporção de partos cesáreos & 0,1034 & 1,9264 & 0,05402 \\
\hline Densidade de população favelada, por km², em 1996 & 0,1040 & 1,9369 & 0,05286 \\
\hline Proporção nascimentos em estabelecimentos privados & 0,1065 & 1,9807 & 0,04765 \\
\hline
\end{tabular}

dimento poderia ter sido interrompido no primeiro passo. Entretanto, com o intuito de explorar um pouco mais a distribuição espacial da variável sob investigação, prosseguimos até o coeficiente padronizado perder a significância no nível de $10 \%$.

No segundo passo, a variável com maior poder explicativo do componente espacial, após o ajuste para a "proporção de mães adolescentes”, foi “proporção de pessoas residentes em favelas em 1996” ( $I=0,0892 ; p=0,09361)$. Após o ajuste da regressão múltipla com as duas variáveis independentes: "proporção de mães adolescentes" e "proporção de pessoas residentes em favelas em 1996", observa-se que ainda é necessário mais um passo, pois o limite crítico de $10 \%$ para o nível de significância não foi alcançado.

No terceiro e último passo, a variável que obteve o maior poder explicativo do compo- nente espacial, depois do ajuste para a "proporção de mães adolescentes" e "proporção de pessoas residentes em favelas em 1996", foi "proporção de chefes com rendimento até um salário mínimo" ( $\mathrm{I}=0,0822 ; \mathrm{p}=0,1200)$. Após o ajuste da regressão múltipla com as três variáveis independentes: "proporção de mães adolescentes”, "proporção de pessoas residentes em favelas em 1996" e "proporção de chefes com rendimento até um salário mínimo”, percebe-se que a autocorrelação espacial dos resíduos não é significativa no nível de $12 \%$, que ultrapassa o limite estabelecido previamente.

As distribuições geográficas da proporção de mães adolescentes e da proporção de chefes com rendimento até um salário mínimo encontram-se representadas, respectivamente, nas Figuras 3 e 4 . Nota-se padrão semelhante para os dois indicadores, com conglomerados 
Tabela 2

Coeficientes de correlação entre os indicadores.

\begin{tabular}{|c|c|c|c|c|c|c|c|c|c|c|}
\hline & $\begin{array}{l}\text { Raiz } \\
\text { quadrada } \\
\text { da taxa de } \\
\text { mortalidade } \\
\text { nos três } \\
\text { primeiros } \\
\text { dias }\end{array}$ & $\begin{array}{l}\text { Log neperia- } \\
\text { no da pro- } \\
\text { porção de } \\
\text { chefes com } \\
\text { rendimento } \\
\text { até um } \\
\text { salário min }\end{array}$ & $\begin{array}{l}\text { Log nepe- } \\
\text { riano da } \\
\text { proporção } \\
\text { de partos } \\
\text { cesáreos }\end{array}$ & $\begin{array}{l}\text { Log nepe- } \\
\text { riano da } \\
\text { proporção } \\
\text { de mães } \\
\text { adolescentes }\end{array}$ & $\begin{array}{l}\text { Log nepe- } \\
\text { riano da } \\
\text { proporção } \\
\text { de mulheres } \\
\text { analfabetas }\end{array}$ & $\begin{array}{l}\text { Raiz qua- } \\
\text { drada da } \\
\text { densidade } \\
\text { de população } \\
\text { favelada, } \\
1996\end{array}$ & $\begin{array}{l}\text { Raiz qua- } \\
\text { drada da } \\
\text { proporção } \\
\text { de pessoas } \\
\text { residentes } \\
\text { em favelas, } \\
1996\end{array}$ & $\begin{array}{l}\text { Raiz qua- } \\
\text { drada do } \\
\text { coeficiente } \\
\text { geral de } \\
\text { natalidade }\end{array}$ & $\begin{array}{l}\text { Proporção } \\
\text { de nasci- } \\
\text { e mentos } \\
\text { em es- } \\
\text { tabele- } \\
\text { cimentos } \\
\text { privados }\end{array}$ & $\begin{array}{l}\text { Proporção de } \\
\text { mães que } \\
\text { não fizeram } \\
\text { pré-natal }\end{array}$ \\
\hline $\begin{array}{l}\text { Raiz quadrada da } \\
\text { taxa de mortalidade } \\
\text { nos três primeiros dia }\end{array}$ & 1,000 & $0,335^{\star \star}$ & $-0,313^{\star \star}$ & $0,412^{\star \star}$ & 0,161 & 0,025 & 0,122 & 0,055 & $-0,150$ & $0,274^{\star \star}$ \\
\hline $\begin{array}{l}\text { Log neperiano da } \\
\text { proporção de chefes } \\
\text { com rendimento até } \\
\text { um salário mínimo }\end{array}$ & $\underbrace{0,335^{\star \star}}$ & 1,000 & $-0,836^{* *}$ & 0,890 ** & $0,669 * *$ & $0,443^{* *}$ & $0,556^{\star *}$ & $0,286^{\star *}$ & $-0,525^{\star *}$ & $0,785^{\star *}$ \\
\hline $\begin{array}{l}\text { Log neperiano } \\
\text { da proporção de } \\
\text { partos cesáreos }\end{array}$ & $-0,313^{* *}$ & $-0,836^{\star *}$ & 1,000 & $-0,826^{\star *}$ & $-0,742^{\star *}$ & $-0,503^{\star *}$ & $-0,585^{\star \star}$ & $-0,309 * *$ & $0,622^{\star *}$ & $-0,819 * *$ \\
\hline $\begin{array}{l}\text { Log neperiano } \\
\text { da proporção de } \\
\text { mães adolescentes }\end{array}$ & $0,412^{\star \star}$ & $0,890 * \star$ & $-0,826 * \star$ & 1,000 & $0,609 * \star$ & $0,412^{\star \star}$ & $0,537^{\star *}$ & $0,316^{\star \star}$ & $-0,558 * \star$ & $0,726 * \star$ \\
\hline $\begin{array}{l}\text { Log neperiano } \\
\text { da proporção de } \\
\text { mulheres analfabetas }\end{array}$ & 0,161 & $0,669 * *$ & $-0,742^{\star \star}$ & $0,609 * *$ & 1,000 & $0,592^{\star \star}$ & $0,792^{\star *}$ & 0,045 & $-0,467^{* *}$ & $0,569 * *$ \\
\hline $\begin{array}{l}\text { Raiz quadrada } \\
\text { da densidade de } \\
\text { população favelada, } \\
1996\end{array}$ & 0,025 & $0,443^{\star \star}$ & $-0,503^{\star \star}$ & $0,412^{\star \star}$ & $0,592^{\star \star}$ & 1,000 & $0,852^{\star \star}$ & $-0,090$ & $-0,543^{\star \star}$ & $0,341^{\star \star}$ \\
\hline $\begin{array}{l}\text { Raiz quadrada } \\
\text { da proporção de } \\
\text { pessoas residentes } \\
\text { em favelas, } 1996\end{array}$ & 0,122 & $0,556^{\star *}$ & $-0,585^{\star \star}$ & $0,537^{\star * \star}$ & $0,792^{\star *}$ & $0,852^{\star *}$ & 1,000 & $-0,056$ & $-0,552^{\star *}$ & $0,379 * *$ \\
\hline $\begin{array}{l}\text { Raiz quadrada do } \\
\text { coeficiente geral } \\
\text { de natalidade }\end{array}$ & 0,055 & $0,286^{\star \star}$ & $-0,309 * \star$ & $0,316^{\star *}$ & 0,045 & $-0,090$ & $-0,056$ & 1,000 & $-0,262^{\star \star}$ & $0,263^{\star *}$ \\
\hline $\begin{array}{l}\text { Proporção de } \\
\text { nascimentos em } \\
\text { estabelecimentos } \\
\text { privados }\end{array}$ & $-0,150$ & $-0,525^{\star \star}$ & $0,622^{\star \star}$ & $-0,558 * \star$ & $-0,467^{\star \star}$ & $-0,543^{\star \star}$ & $-0,552^{\star \star}$ & $-0,262^{\star \star}$ & 1,000 & $-0,360 * \star$ \\
\hline $\begin{array}{l}\text { Proporção de mães } \\
\text { que não fizeram } \\
\text { pré-natal }\end{array}$ & $0,274^{\star *}$ & $0,785^{\star \star}$ & $-0,819 * \star$ & $0,726^{\star \star *}$ & $0,569 * \star$ & 0,341 ** & $0,379 * *$ & $0,263^{\star \star}$ & $-0,360 * \star$ & 1,000 \\
\hline
\end{tabular}

** Correlação é significativa no nível 0,01. 


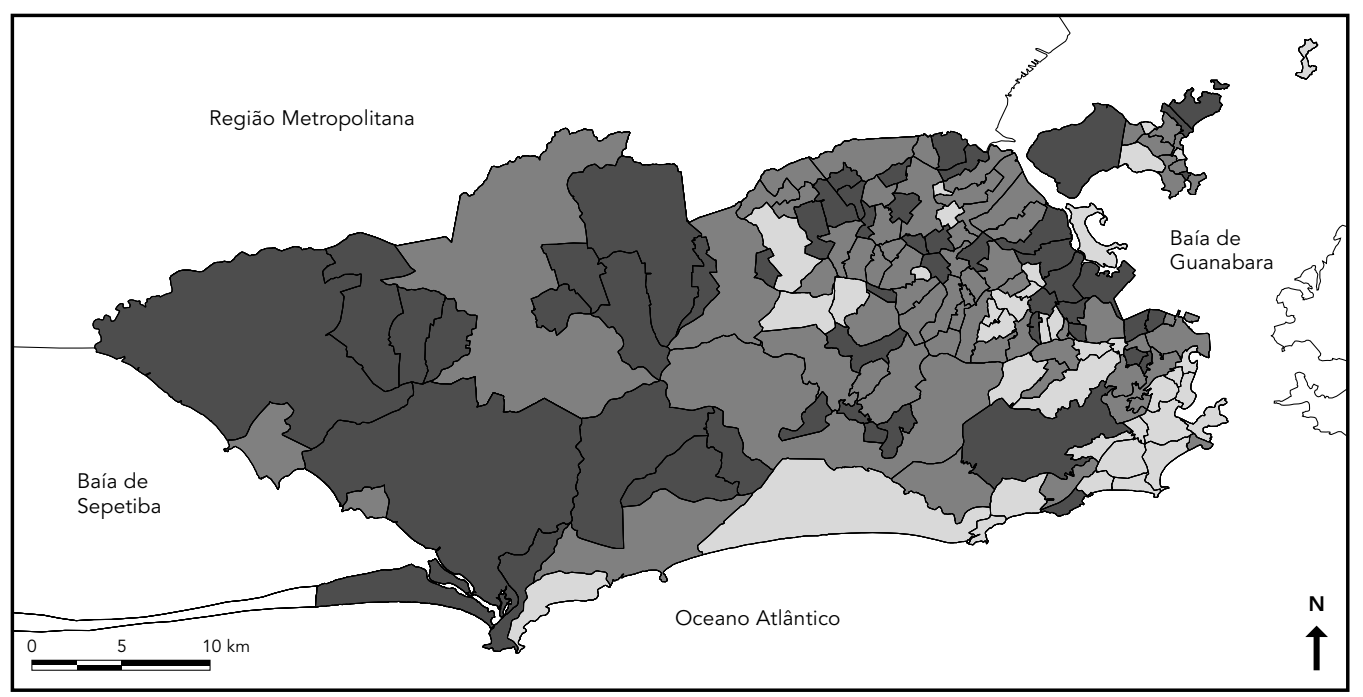

$$
\begin{aligned}
& \text { até } 14,0 \\
& 14,1 \text { a } 20,5 \\
& 20,6 \text { e mais }
\end{aligned}
$$

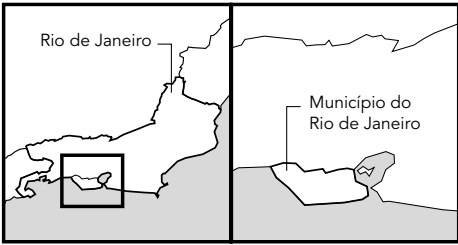

Fonte: Secretaria Municipal de Saúde do Rio de Janeiro e Sistema de Informação Geográfica/Fundação Oswaldo Cruz.

de taxas elevadas na Zona Oeste e parte da Zona Norte do Município do Rio de Janeiro, evidenciando-se padrão similar ao da taxa de mortalidade nos três primeiros dias de vida.

\section{Discussão}

Análise espacial, campo da Estatística que estuda quantitativamente fenômenos que se manifestam no espaço, tem como uma de suas aplicações a análise exploratória de dados epidemiológicos (Mayer, 1983). A primeira etapa deste tipo de análise consiste em pesquisar a ocorrência de autocorrelação espacial. Quando se verifica que há dependência espacial, a lógica posterior da análise é identificar as variáveis explicativas dos conglomerados geográficos encontrados, que se constituem no conjunto dos possíveis fatores de risco.

Sob esta óptica, vários estudos no nível ecológico têm mostrado contribuições relevantes à pesquisa epidemiológica, na medida em que apontam caminhos para investigar potenciais associações com as variações espaciais da ocorrência da doença. Grande parte desses estudos têm sido baseados no coeficiente de autocorrelação proposto inicialmente por Moran (1950) e generalizado por Cliff \& Ord (1981), apesar de suas limitações quando existe alta heterogeneidade da densidade populacional entre as subáreas geográficas (Assunção \& Reis, 1999).

Andes \& Davis (1995) evidenciaram a importância dos estudos de análise espacial para identificar contextos geográficos importantes na explicação das variações da mortalidade infantil no Alasca. Os autores concluem que as técnicas utilizadas foram fundamentais para descrever as condições geográficas da mortalidade infantil, e que os achados contribuíram relevantemente para sugerir hipóteses a serem investigadas posteriormente.

No presente trabalho, estabeleceu-se primeiramente a existência de dependência espa- 


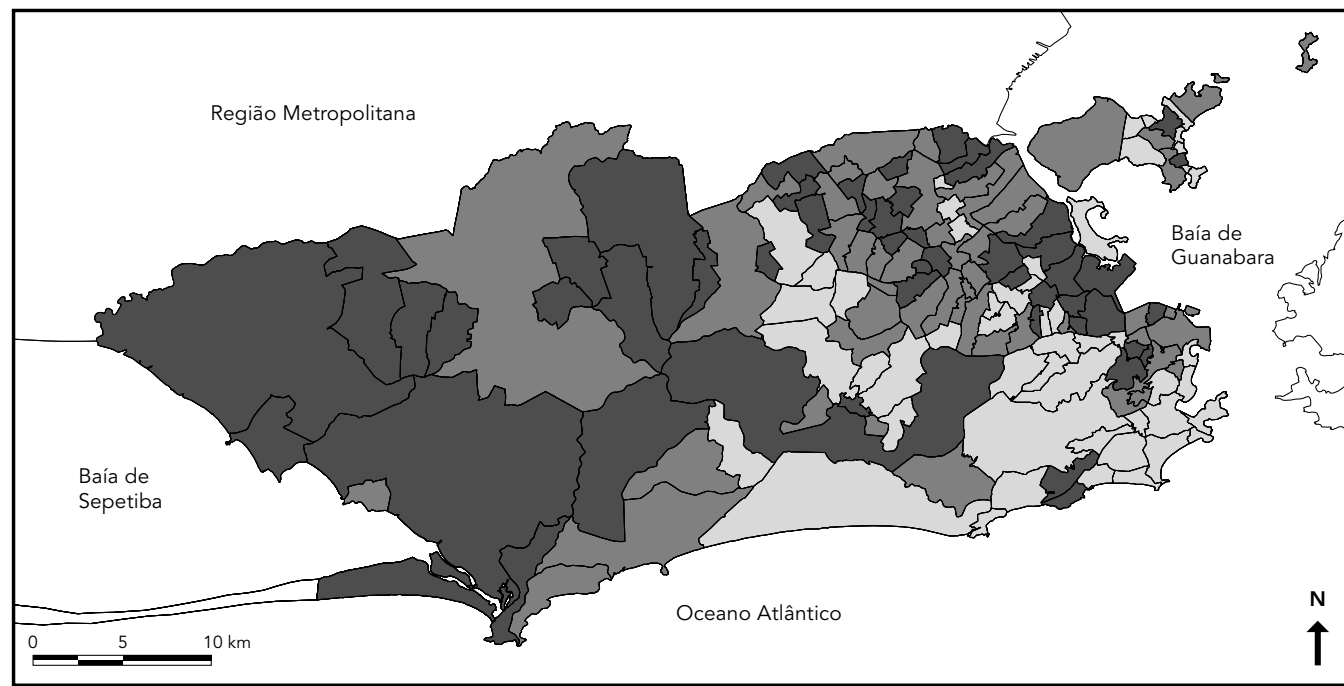

$$
\begin{aligned}
& \text { até } 15,85 \\
& 15,86 \text { a } 24,13 \\
& 24,14 \text { e mais }
\end{aligned}
$$

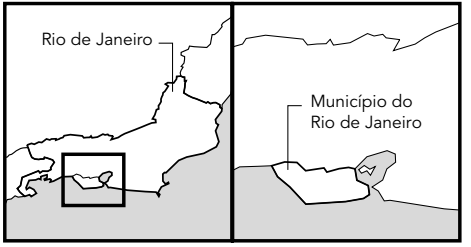

Fonte: IBGE, 1991.

cial na taxa de mortalidade nos três primeiros dias de vida. Isto significa que a distribuição geográfica deste indicador não é aleatória, ou seja, existem conglomerados de bairros onde a taxa de mortalidade nos três primeiros dias de vida é elevada em relação aos outros bairros.

Em uma segunda etapa, procurou-se estabelecer, através de procedimento passo a passo, as variáveis que melhor explicassem o componente espacial. A variável mais explicativa foi a "proporção de mães adolescentes", que sozinha foi responsável pela perda de significância da autocorrelação espacial no nível de significância de 5\%. Este indicador foi também o que mostrou a maior correlação com a taxa de mortalidade nos três primeiros dias de vida.

Nos passos seguintes do procedimento de escolha das variáveis, a "proporção de pessoas residentes em favelas" seguida da "proporção de chefes com rendimento até um salário mínimo" foram os outros dois indicadores selecionados, sugerindo que as variações espaciais da mortalidade nos três primeiros dias são explicadas, em parte, pela concentração residencial da pobreza.

Como comentado por Massey (1996), o fato de a pobreza estar geograficamente agregada tem profundas implicações para a natureza da vida social. A concentração de jovens residentes em comunidades carentes, como as favelas, tem sido associada ao bem-estar emocional e aos comportamentos ditos "de risco" que influenciam a sua saúde, como o uso de drogas e álcool, a violência, as infecções sexualmente transmissíveis e a gravidez precoce entre as meninas adolescentes (Sells \& Blum, 1996).

A literatura internacional aponta para resultados compatíveis aos nossos. Em estudo ecológico na Inglaterra, Slogget \& Joshi (1998) estudaram a associação entre o grau de privação social e alguns agravos de saúde. Os resultados mostraram forte associação com a gravidez na adolescência.

Boulton-Jones et al. (1995), em estudo ecológico na Escócia, analisaram as relações entre 
as taxas de concepção entre adolescentes e o nível de pobreza da área de residência. Foram evidenciadas enormes diferenças nas taxas de concepção, de 4 a 5 vezes maior entre as que viviam em situação de grande privação social quando comparadas às que residiam nas áreas mais ricas.

Em estudo espacial das taxas de fecundidade de adolescentes de 15 a 17 anos de idade na Califórnia, Estados Unidos, Gould et al. (1998) enfatizaram a importância de investigar as diferenças entre pequenas áreas geográficas. Foram encontradas variações relevantes nos níveis de pobreza e educação assim como nos cuidados pré-natais.

A associação entre a idade jovem da mãe e resultados adversos do recém-nascido tem sido objeto de pesquisa recente (Fraser et al., 1995; Roth et al.,1998). Os achados levam a concluir que, embora os fatores de natureza biológica sejam importantes - tais como a imaturidade do sistema reprodutivo e o ganho inadequado de peso durante a gravidez -, a falta de atendimento pré-natal das adolescentes, associada à pobreza e níveis baixos de instrução, tem desempenhado papel preponderante na morbi-mortalidade dos recém-nascidos.

Sastry (1996), ao analisar dados de mortalidade na infância no Brasil, procurou explicar, dentre seus objetos de estudo, como o contexto ambiental e social em que a criança reside afeta o seu risco de morrer, buscando entender como as características do domicílio modificam os efeitos coletivos da comunidade. Embora as características da comunidade de residência tenham mostrado associações relevantes com o risco de morrer na infância, os efeitos foram, em geral, mediados pelos atributos da mãe, como, por exemplo, o nível materno de educação.

Os resultados da nossa análise apontaram para expressivas correlações entre a falta de atenção pré-natal, a proporção de mães ado- lescentes, o nível de educação e a concentração de pobreza do bairro de residência. Podemos, assim, hipotetizar que a mortalidade infantil sofre a influência de fatores em níveis distintos, tanto individuais - como os relacionados ao comportamento da adolescente em relação à gestação, ao seu estilo de vida, ao seu grau de instrução - quanto coletivos - relacionados ao grau de privação social e à marginalidade da comunidade em que reside.

A investigação dessa hipótese, no entanto, requer o uso de outras metodologias que lidem com informações agregadas em diferentes níveis, o individual e o coletivo, tal como a modelagem em múltiplos níveis (Goldstein, 1995; Hox, 1995), que será objeto de pesquisa em futuro próximo.

Para finalizar, vale dizer que o uso da metodologia estatística espacial na nossa análise permitiu maior compreensão da distribuição geográfica da mortalidade neonatal precoce no Município do Rio de Janeiro, identificando os problemas relacionados à gravidez em adolescentes e à marginalidade social como as questões mais relevantemente associadas.

No que se refere ao procedimento passo a passo introduzido na análise, apesar de apresentar a mesma restrição dos procedimentos stepwise de regressão múltipla, ou seja a de realizar múltiplos testes de hipóteses com um nível de significância único, pode-se dizer que a sua aplicação mostrou resultados importantes e coerentes com os achados na literatura. A seleção das variáveis mais explicativas dos conglomerados espaciais possibilitou identificar potenciais fatores associados ao risco do recém-nascido de morrer nos primeiros dias de vida, que poderão dar margens a investigações mais analíticas, tanto no nível individual como no coletivo, e subsidiar os programas preventivos, contribuindo ao declínio da mortalidade infantil no município. 


\section{Referências}

ANDES, N. \& DAVIS, J. E., 1995. Linking public health data using geographic information system techniques: Alaskan community characteristics and infant mortality. Statistics in Medicine, 14:481-490.

ANSELIN, L., 1992. SpaceStat Tutorial. A Workbook for Using SpaceStat in the Analysis of Spatial Data. Morgantown: Regional Research Institute, West Virginia University.

ASSUNÇÃO, R. M. \& REIS, E., 1999. A new proposal to adjust Moran's I for population density. Statistics in Medicine, 18:2147-2162.

BOULTON-JONES, C.; McILWAINE, G. \& McINNENY, K., 1995. Teenage pregnancy and deprivation. BMJ, 310:398-399.

CLIFF, A. D. \& ORD, J. K., 1981. Spatial Process: Models and Applications. London: Pion.

CORRÊA, R. L., 1996. Trajetórias Geográficas. Rio de Janeiro: Bertrand Brasil.

FRASER, A. M.; BROCKERT, J. E. \& WARD, R. H., 1995. Association of young maternal age with adverse reproductive outcomes. New England Journal of Medicine, 332:1114-1117.

GEARY, R. C., 1954. The contiguity ratio and statistical mapping. Incorporated Statistian, 5:115-145.

GOLDSTEIN, H., 1995. Multilevel Statistical Models. London: Edward Arnold.

GOULD, J. B.; HERRCHEN, B.; PHAN, T.; BERA, S. \& BRINDIS, C., 1998. Small-area analysis: Targeting high-risk areas for adolescent pregnancy prevention programs. Family Planning Perspectives, 30: 173-176.

HOX, J. J., 1995. Applied Multilevel Analysis. Amsterdam: TT-Publikaties.

IBGE (Fundação Instituto Brasileiro de Geografia e Estatística), 1994. Censo Demográfico. Brasil, 1991. Rio de Janeiro: IBGE.

ISSLER, R. M. S.; GIUGLIANI, E. R. J.; KREUTZ, G. T.; MENESES, C. F.; JUSTO, E. B.; KREUTZ, V. M. \& PIRES, M., 1996. Poverty levels and children's health status: Study of risk factors in an urban population of low socioeconomic level. Revista de Saúde Pública, 30:506-511.

KAPLAN, G. A., 1996. People and places: Contrasting perspectives on the association between social class and health. International Journal of Health Services, 26:507-519.

KAWACHI, I. \& KENNEDY, B. P., 1997. Health and social cohesion: Why care about income inequality? $B M J, 314: 1037-1040$

LEAL, M. C. \& SZWARCWALD, C. L., 1996. Evolução da mortalidade neonatal no Estado do Rio de Janeiro, Brasil, de 1979 a 1993. 1 - Análise por grupo etário segundo região de residência. Revista de Saúde Pública, 30:403-412.

MAPINFO CORPORATION, 1994. MapInfo Reference. New York: MapInfo Corporation.

MASSEY, D. S., 1996. The age of extremes: Concentrated affluence and poverty in the twenty-first century. Demography, 33:395-412.

MATHSOFT INCORPORATION, 1996. S+SPATIALSTATS User's Manual, Version 1.0. Seattle: MathSoft Inc.

MAYER, J. D., 1983. The role of spatial analysis and geographic data in the detection of disease causation. Social Science and Medicine, 17:1212-1221.
MENEZES, A. M. B.; BARROS, F. C.; VICTORA, C. G.; TOMASI, E.; HALPERN, R. \& OLIVEIRA, A. L. B., 1998. Fatores de risco para mortalidade perinatal em Pelotas, RS, 1993. Revista de Saúde Pública, 32:209-216

MENEZES, A. M. B.; VICTORA, C. G; BARROS, F. C.; ALBERNAZ, E.; MENEZES, F. S.; JANNKE, H. A.; ALVES, C. \& ROCHA, C., 1996. Mortalidade infantil em duas coortes de base populacional no Sul do Brasil: Tendências e diferenciais. Cadernos de Saúde Pública, 12(Sup.):79-86.

MORAN, P. A. P., 1950. Notes on continuous stochastic phenomena. Biometrika, 37:17-23.

RIP, M. R.; KEEN, C. S. \& WOODS, D. L., 1987. Intraurban variations of neonatal and post-neonatal mortality in a developing city. Social Science and Medicine, 25:889-894.

ROTH, J.; HENDRICKSON, J.; SCHILLING, M. \& STOWELL, D. W., 1998. The risk of teen mothers having low birth weight babies: Implications of recent medical research for school health personnel. Journal of School Health, 68:271-275.

SASTRY, N., 1996. Community characteristics, individual and household attributes, and child survival in Brazil. Demography, 33:211-229.

SSMA-RS (Secretaria da Saúde e do Meio Ambiente do Rio Grande do Sul), 1998. Mortalidade infantil no Rio Grande do Sul. Boletim Epidemiológico, no 5. Porto Alegre: SSMA-RS.

SELLS, C. W. \& BLUM, R. W., 1996. Morbidity and mortality among US adolescents: An overview of data and trends. American Journal of Public Health, 86:513-519.

SEN, A., 1990. Distribution of Spatial Correlation Statistics. Spatial Statistics: Past, Present and Future. New York: Institute of Mathematical Geography.

SLOGGETT, A. \& JOSHI, H., 1998. Deprivation indicators as predictors of life events 1981-1992 based on the UK ONS longitudinal study. Journal of Epidemiology and Community Health, 52:228-233.

SPSS INCORPORATION, 1995. SPSS for Windows. Stastical Package for the Social Sciences. Release 7.0. Chicago: SPSS Inc.

WILKINSON, R. G., 1997. Comment: Income, inequality, and social cohesion. American Journal of Public Health, 87:1504-1506. 